\title{
A study on Ricci soliton in $S$-manifolds.
}

\author{
K.R. Vidyavathi and C.S. Bagewadi \\ Department of Mathematics, Kuvempu University,Shankaraghatta - 577 451, Shimoga, Karnataka, India.
}

\begin{abstract}
In this paper, we study semi symmetric and pseudo symmetric conditions in $S$-manifolds, those are $R \cdot R=0 \quad, \quad R \cdot C=0 \quad, \quad C \cdot R=0 \quad, \quad C \cdot C=0 \quad, \quad R \cdot R=L_{1} Q(g, R) \quad, \quad R \cdot C=L_{2} Q(g, C)$, $C \cdot R=L_{3} Q(g, R)$, and $C \cdot C=L_{4} Q(g, C)$, where $C$ is the Concircular curvature tensor and $L_{1}, L_{2}, L_{3}, L_{4}$ are the smooth functions on $M$, further we discuss about Ricci soliton.
\end{abstract}

Keywords: $S$-manifold, $\eta$-Einstein manifold, Einstein manifold, Ricci soliton.

\section{Introduction}

The notion of $f$-structure on a $(2 n+s)$-dimensional manifold $M$, i.e., a tensor field of type $(1,1)$ on $M$ of rank $2 n$ satisfying $f^{3}+f=0$, was firstly introduced in 1963 by K. Yano [28] as a generalization of both (almost) contact (for $\mathrm{s}=1$ ) and (almost) complex structures (for $\mathrm{s}=0$ ). During the subsequent years, this notion has been furtherly developed by several authors [3], [4], [11], [12], [15], [16], [17]. Among them, H. Nakagawa in [16] and [17] introduced the notion of framed $f$-manifold, later developed and studied by S.I. Goldberg and K. Yano ([11], [12]) and others with the denomination of globally framed $f$-manifolds.

A Riemannian manifold $M$ is called locally symmetric if its curvature tensor $R$ is parallel, i.e., $\nabla R=0$, where $\nabla$ denotes the Levi-Civita connection. As a generalization of locally symmetric manifolds the notion of semisymmetric manifolds was defined by

$(R(X, Y) \cdot R)(U, V) W=0, X, Y, U, V, W \in T M$

and studied by many authors [18], [19], [26], [20]. Z.I. Szabo [25] gave a full intrinsic classification of these spaces. R. Deszcz $[8,9]$ weakened the notion of semisymmetry and introduced the notion of pseudosymmetric manifolds by

$$
(R(X, Y) \cdot R)(U, V) W=L_{R}[((X \wedge Y) \cdot R)(U, V) W],
$$

where $L_{R}$ is smooth function on $M$ and $X \wedge Y$ is an endomorphism defined by

$$
(X \wedge Y) Z=g(Y, Z) X-g(X, Z) Y .
$$

Definition 1 A Ricci soliton is a natural generalization of an Einstein metric and is defined on a Riemannian manifold $(M, g)$. A Ricci soliton is a triple $(g, V, \lambda)$ with $g$ is a Riemannian metric, $V$ is a vector field and $\lambda$ is a real scalar such that

$$
\left(L_{V}\right) g(X, Y)+2 S(X, Y)+2 \lambda g(X, Y)=0 \text {. }
$$

where $S$ is a Ricci tensor of $M$ and $L_{V}$ denotes the Lie derivative operator along the vector field $V$

. The Ricci soliton is said to be shrinking, steady and expanding according as $\lambda$ is negative, zero and positive respectively. The authors R.Sharma [22, 23, 24] and M.M.Tripathi [27] initiated the study of Ricci solitons in contact manifold. But Călin and Crasmareanu [6], Bagewadi and Ingalahalli [14, 1], S.Debnath and A.Battacharya [7] have studied the existence and also obtained results on Ricci solitons in $f$-kenmotsu manifolds, $\alpha$-Sasakian manifolds, Lorentzian $\alpha$-Sasakian manifolds, Trans-Sasakian manifolds using L.P.Eisenhart problem [10]. But C.S.Bagewadi, Ingalahalli and Ashok, C.S.Bagewadi and K.R.Vidyavathi have studied Ricci solitons in Kenmotsu manifolds, almost $C(\alpha)$ manifolds using semi-symmetric and pseudosymmetric conditions [2]. In the present paper, we study Ricci soliton in $S$-manifolds satisfying semi symmetric and pseudo symmetric conditions those are $R \cdot R=0, R \cdot C=0, C \cdot R=0, C \cdot C=0$, $R \cdot R=L_{1} Q(g, R), R \cdot C=L_{2} Q(g, C), C \cdot R=L_{3} Q(g, R)$, and $C \cdot C=L_{4} Q(g, C)$, where $C$ is the 
Concircular curvature tensor and $L_{1}, L_{2}, L_{3}, L_{4}$ are the smooth functions on $M$.

\section{Preliminaries}

Let $M$ be a $(2 n+s)$-dimensional manifold with an $f$-structure of rank $2 n$. If there exists global vector fields $\xi_{\alpha}, \alpha=(1,2,3, \ldots \ldots, s)$ on $M$ such that;

$$
\begin{aligned}
& f^{2}=-I+\sum \xi_{\alpha} \otimes \eta_{\alpha}, \quad \eta_{\alpha}\left(\xi_{\beta}\right)=\delta_{\beta}^{\alpha}, \quad(2.1) \\
& f \xi_{\alpha}=0, \eta_{\alpha} \circ f=0, \quad(2.2) \\
& g\left(X, \xi_{\alpha}\right)=\eta_{\alpha}(X), \quad g(X, f Y)=-g(f X, Y),
\end{aligned}
$$

where $\eta_{\alpha}$ are the dual 1-forms of $\xi_{\alpha}$, we say that the $f$-structure has complemented frames. For such a manifold there exists a Riemannian metric $g$ such that

$$
g(X, Y)=g(f X, f Y)+\sum \eta_{\alpha}(X) \eta_{\alpha}(Y)
$$

for any vector fields $X$ and $Y$ on $M$.

An $f$-structure $f$ is normal, if it has complemented frames and

$$
[f, f]+2 \sum \xi_{\alpha} \otimes d \eta_{\alpha}=0
$$

where $[f, f]$ is Nijenhuis torsion of $f$.

Let $F$ be the fundamental 2-form defined by $F(X, Y)=g(X, f Y), X, Y \in T(M)$. A normal $f$-structure for which the fundamental form $F$ is closed, $\eta_{1} \wedge, \ldots \ldots, \eta_{s} \wedge\left(d \eta_{\alpha}\right)^{n} \neq 0$ for any $\alpha$, and $d \eta_{1}=\ldots .=d \eta_{s}=F$ is called to be an $S$-structure. A smooth manifold endowed with an $S$-stucture will be called an $S$-manifold. These manifolds introduced by Blair [3].

We have to remark that if we take $s=1, S$-manifolds are natural generalizations of Sasakian manifolds. In the case $s \geq 2$ some interesting examples are given [3], [13].

If $M$ is an $S$-manifold, then the following relations holds true [3];

$$
\begin{aligned}
& \nabla_{X} \xi_{\alpha}=-f X, \quad X \in T(M), \alpha=1,2, \ldots, s \\
& \left(\nabla_{X} f\right) Y=\sum\left\{g(f X, f Y) \xi_{\alpha}+\eta_{\alpha}(Y) f^{2} X\right\}, X, Y \in T(M),
\end{aligned}
$$

where $\nabla$ is the Riemannian connection of $g$. Let $\Omega$ be the distribution determined by the projection tensor$f^{2}$ and let $N$ be the complementry distribution which is determined by $f^{2}+I$ and spanned by $\xi_{1}, \ldots . . \xi_{s}$. It is clear that if $X \in \Omega$ then $\eta_{\alpha}(X)=0$ for any $\alpha$, and if $X \in N$, then $f X=0$. A plane section $\pi$ on $M$ is called an invariant $f$-section if it is determined by a vector $X \in \Omega(x), x \in M$, such that $\{X, f X\}$ is an orthonormal pair spanning the section. The sectional curvature of $\pi$ is called the $f$-sectional curvature. If $M$ is an $S$-manifold of constant $f$-sectional curvature $k$, then its curvature tensor has the form

$$
\begin{aligned}
R(X, Y, Z, W)=\sum_{\alpha, \beta}\left\{g(f X, f W) \eta_{\alpha}(Y) \eta_{\beta}(Z)-g(f X, f Z) \eta_{\alpha}(Y) \eta_{\beta}(W)+g(f Y, f Z) \eta_{\alpha}(X) \eta_{\beta}(W)\right. \\
\left.\quad-g(f Y, f W) \eta_{\alpha}(X) \eta_{\beta}(Z)\right\}+\frac{1}{4}(k+3 s)\{g(f X, f W) g(f Y, f Z)-g(f X, f Z) g(f Y, f W)\} \\
+\frac{1}{4}(k-s)\{F(X, W) F(Y, Z)-F(X, Z) F(Y, W)-2 F(X, Y) F(Z, W)\},
\end{aligned}
$$

where $X, Y, Z, W \in T(M)$. Such a manifold $N(K)$ will be called an $S$-space form. The Euclidean space $E^{2 n+s}$ and the hiperbolic space $H^{2 n+s}$ are examples of $S$-space forms. 
Definition $2 S$-manifold $\left(M, f, \eta_{\alpha}, g, \xi_{\alpha}\right)$ is said to be $\eta$-Einstein if the Ricci tensor $S$ of $M$ is of the form

$$
S=a g+b \sum_{\alpha=1}^{s} \eta_{\alpha} \otimes \eta_{\alpha}
$$

where $a, b$ are constants on $\mathrm{M}$.

Now contracting equation (2.7) we get

$S(Y, Z)=\left[\frac{4 s+(k+3 s)(2 n-1)+3(k-s)}{4}\right] g(Y, Z)+\left[\frac{(2 n+s-2)(4-k-3 s)-3(k-s)}{4}\right] \sum_{\alpha} \eta_{\alpha}(Y) \eta_{\alpha}(Z)$,

$S\left(Y, \xi_{\alpha}\right)=\frac{1}{4}\left[s^{2}(13-6 n-k-3 s)+2 s(7 n-5)+k(2-s)+2 n k(1-s)\right]$.

From (2.7) we have

$$
\begin{aligned}
& R(X, Y) \xi_{\alpha}=s \sum_{\alpha}\left\{\eta_{\alpha}(Y) X-\eta_{\alpha}(X) Y\right\} \\
& R\left(\xi_{\alpha}, Y\right) Z=s \sum_{\alpha}\left\{g(Y, Z) \xi_{\alpha}-\eta_{\alpha}(Z) Y\right\} \\
& \eta_{\alpha}(R(X, Y) Z)=s \sum_{\alpha}\left\{g(Y, Z) \eta_{\alpha}(X)-g(X, Z) \eta_{\alpha}(Y)\right\}
\end{aligned}
$$

\section{Ricci Soliton In Semi-Symmetric $S$-Manifolds}

An $S$-manifold is said to be semi-symmetric if $R \cdot R=0$.

$$
\begin{aligned}
& \left(R\left(\xi_{\alpha}, Y\right) \cdot R\right)(U, V) W=0, \\
& R\left(\xi_{\alpha}, Y\right) R(U, V) W-R\left(R\left(\xi_{\alpha}, Y\right) U, V\right) W-R\left(U, R\left(\xi_{\alpha}, Y\right) V\right) W-R(U, V) R\left(\xi_{\alpha}, Y\right) W=0 .
\end{aligned}
$$

Using (2.11) in (3.2), we get

$$
\begin{aligned}
& s \sum_{\alpha}\left\{g(Y, R(U, V) W) \xi_{\alpha}-\eta_{\alpha}(R(U, V) W) Y-g(Y, U) R\left(\xi_{\alpha}, V\right) W+\eta_{\alpha}(U) R(Y, V) W\right. \\
& \left.-g(Y, V) R\left(U, \xi_{\alpha}\right) W+\eta_{\alpha}(V) R(U, Y) W-g(Y, W) R(U, V) \xi_{\alpha}+\eta_{\alpha}(W) R(U, V) Y\right\}=0
\end{aligned}
$$

By taking an inner product with $\xi_{\alpha}$ then we get

$\sum_{\alpha}\left\{s R(U, V, W, Y)-\eta_{\alpha}(R(U, V) W) \eta_{\alpha}(Y)-g(Y, U) \eta_{\alpha}\left(R\left(\xi_{\alpha}, V\right) W\right)+\eta_{\alpha}(U) \eta_{\alpha}(R(Y, V) W)\right.$

$\left.-g(Y, V) \eta_{\alpha}\left(R\left(U, \xi_{\alpha}\right) W\right)+\eta_{\alpha}(V) \eta_{\alpha}(R(U, Y) W)-g(Y, W) \eta_{\alpha}\left(R(U, V) \xi_{\alpha}\right)+\eta_{\alpha}(W) \eta_{\alpha}(R(U, V) Y)\right\}=0$.

By using (2.10), (2.12) in (3.4) we have

$$
s R(U, V, W, Y)+s^{2} g(Y, V) g(U, W)-s^{2} g(Y, U) g(V, W)=0 . \text { (3.5) }
$$

Taking $U=Y=e_{i}$ in (3.5) and summing over $i=1,2, \ldots \ldots .2 n+s$ we get

$$
S(V, W)=s(2 n+s-1) g(V, W)
$$

Thus we state the following;

Theorem 1 Semi symmetric $S$-manifold is an Einstein manifold.

If $V$ is co-linear with $\xi$, then Ricci soliton along $\xi$ is given by

$$
\left(L_{\xi} g\right)(X, Y)+2 S(X, Y)+2 \lambda g(X, Y)
$$

Definition 3 Let $\left(f, \xi_{1}, \xi_{2}, \ldots \ldots, \xi_{s}, \eta_{1}, \eta_{2}, \ldots \ldots, \eta_{s}, g\right)$ is the contact $S$-frame manifold, if $V$ is in the linear span (combination) of $\xi_{1}, \xi_{2}, \ldots \ldots \xi_{s}$ then $V=c_{1} \xi_{1}+c_{2} \xi_{2}+\ldots \ldots \ldots . .+c_{s} \xi_{s}$ and the Ricci soliton is a 
triple $\left(g, \xi_{\alpha}, \lambda\right)$ with $g$ is a Riemannian metric, $\xi_{\alpha},(\alpha=1,2, \ldots s)$ is a vector field and $\lambda$ is a real scalar such that

$$
\left(\sum_{i=1}^{s} c_{i} L_{\xi_{i}} g\right)(X, Y)+2 S(X, Y)+2 \lambda g(X, Y)=0
$$

From (3.7) we have

$$
c_{i} g\left(\nabla_{X} \xi_{\alpha}, Y\right)+c_{i} g\left(\nabla_{Y} \xi_{\alpha}, X\right)+2 S(X, Y)+2 \lambda g(X, Y)=0 .
$$

Using (2.5) in (3.8) we get

$$
c_{i} g(-f X, Y)+c_{i} g(-f Y, X)+2 S(X, Y)+2 \lambda g(X, Y)=0
$$

From (3.6) and (3.9) we have

$$
(s(2 n+s-1)+\lambda) g(X, Y)=0(3.10)
$$

Taking $X=Y=e_{i}$ in (3.10) and summing over $i=1,2, \ldots \ldots .2 n+s$, we get the value of $\lambda$

Thus we state the following;

$$
\lambda=-s(2 n+s-1)(<0)
$$

Theorem 2 Ricci soliton in semi-symmetric $S$-manifold is shrinking.

Corollary 1 Ricci soliton in semi symmetric $S$-manifold is steady if $s=0$ (Kaehler manifold) and is shrinking if $s=1$ (Sasakian manifold).

IV. Ricci soliton in $S$-manifolds satisfying $R \cdot C=0$.

The Concircular curvature tensor $\mathrm{C}$ is given by

$$
C(X, Y) Z=R(X, Y) Z-\frac{r}{2 n(2 n+1)}\{g(Y, Z) X-g(X, Z) Y\}
$$

Using (2.10), (2.11) and (2.12) in (4.1) we get

$$
\begin{aligned}
& C(X, Y) \xi_{\alpha}=\left[s-\frac{r}{2 n(2 n+1)}\right] \sum_{\alpha}\left\{X \eta_{\alpha}(Y)-\eta_{\alpha}(X) Y\right\} \\
& C\left(\xi_{\alpha}, Y\right) Z=\left[s-\frac{r}{2 n(2 n+1)}\right] \sum_{\alpha}\left\{g(Y, Z) \xi_{\alpha}-Y \eta_{\alpha}(Z)\right\} \\
& \eta_{\alpha}(C(X, Y) Z)=\left[s-\frac{r}{2 n(2 n+1)}\right] \sum_{\alpha}\left\{g(Y, Z) \eta_{\alpha}(X)-g(X, Z) \eta_{\alpha}(Y)\right\}
\end{aligned}
$$

Let us assume that the condition $R\left(\left(\xi_{\alpha}, Y\right) \cdot C\right)(U, V) W=0$ hold on $M$, then

$R\left(\xi_{\alpha}, Y\right) C(U, V) W-C\left(R\left(\xi_{\alpha}, Y\right) U, V\right) W-C\left(U, R\left(\xi_{\alpha}, Y\right) V\right) W-C(U, V) R\left(\xi_{\alpha}, Y\right) W=0$.

Using (2.11) in (4.5), we get

$$
\begin{aligned}
& s \sum_{\alpha}\left\{g(Y, C(U, V) W) \xi_{\alpha}-\eta_{\alpha}(C(U, V) W) Y-g(Y, U) C\left(\xi_{\alpha}, V\right) W+\eta_{\alpha}(U) C(Y, V) W\right. \\
& \left.-g(Y, V) C\left(U, \xi_{\alpha}\right) W+\eta_{\alpha}(V) C(U, Y) W-g(Y, W) C(U, V) \xi_{\alpha}+\eta_{\alpha}(W) C(U, V) Y\right\}=0
\end{aligned}
$$

By taking an inner product with $\xi_{\alpha}$ then we get

$$
\begin{aligned}
& \sum_{\alpha}\left\{s C(U, V, W, Y)-\eta_{\alpha}(C(U, V) W) \eta_{\alpha}(Y)-g(Y, U) \eta_{\alpha}\left(C\left(\xi_{\alpha}, V\right) W\right)+\eta_{\alpha}(U) \eta_{\alpha}(C(Y, V) W)\right. \\
& \left.-g(Y, V) \eta_{\alpha}\left(C\left(U, \xi_{\alpha}\right) W\right)+\eta_{\alpha}(V) \eta_{\alpha}(C(U, Y) W)-g(Y, W) \eta_{\alpha}\left(C(U, V) \xi_{\alpha}\right)+\eta_{\alpha}(W) \eta_{\alpha}(C(U, V) Y)\right\}=0 .
\end{aligned}
$$

By using (4.2), (4.4) in (4.7) we have 
$C(U, V, W, Y)=\left[s-\frac{r}{2 n(2 n+1)}\right]\{g(Y, U) g(V, W)-g(Y, V) g(U, W)\}$.

Taking $U=Y=e_{i}$ in (4.8) and summing over $i=1,2, \ldots \ldots . .2 n+s$ and using (4.1) we get

$$
S(V, W)=s(2 n+s-1) g(V, W)
$$

Thus we state the following;

Theorem $3 S$-manifold satisfying the condition $R \cdot C=0$ is an Einstein manifold.

From (4.9) and (3.9) we have

$$
(s(2 n+s-1)+\lambda) g(X, Y)=0(4.10)
$$

Taking $X=Y=e_{i}$ in (4.10) and summing over $i=1,2, \ldots \ldots .2 n+s$, we get the value of $\lambda$

$$
\lambda=-s(2 n+s-1)(<0)
$$

Thus we state the following;

Theorem 4 Ricci soliton in $S$-manifold satisfying the condition $R \cdot C=0$ is shrinking.

Corollary 2 Ricci soliton in $S$-manifold satisfying $R \cdot C=0$ is steady if $s=0$ (Kaehler manifold) and is shrinking if $s=1$ (Sasakian manifold).

\section{Ricci soliton in $S$-manifolds satisfying $C \cdot R=0$.}

Let us assume that the condition $C\left(\left(\xi_{\alpha}, Y\right) \cdot R\right)(U, V) W=0$ hold on $M$, then

$$
C\left(\xi_{\alpha}, Y\right) R(U, V) W-R\left(C\left(\xi_{\alpha}, Y\right) U, V\right) W-R\left(U, C\left(\xi_{\alpha}, Y\right) V\right) W-R(U, V) C\left(\xi_{\alpha}, Y\right) W=0 .
$$

Using (4.3) in (5.1), we get

$$
\begin{gathered}
{\left[s-\frac{r}{2 n(2 n+1)}\right] \sum_{\alpha}\left\{g(Y, R(U, V) W) \xi_{\alpha}-\eta_{\alpha}(R(U, V) W) Y-g(Y, U) R\left(\xi_{\alpha}, V\right) W+\eta_{\alpha}(U) R(Y, V) W\right.} \\
\left.-g(Y, V) R\left(U, \xi_{\alpha}\right) W+\eta_{\alpha}(V) R(U, Y) W-g(Y, W) R(U, V) \xi_{\alpha}+\eta_{\alpha}(W) R(U, V) Y\right\}=0
\end{gathered}
$$

By taking an inner product with $\xi_{\alpha}$ then we get

$$
\begin{aligned}
& \sum_{\alpha}\left\{s R(U, V, W, Y)-\eta_{\alpha}(R(U, V) W) \eta_{\alpha}(Y)-g(Y, U) \eta_{\alpha}\left(R\left(\xi_{\alpha}, V\right) W\right)+\eta_{\alpha}(U) \eta_{\alpha}(R(Y, V) W)\right. \\
& \left.-g(Y, V) \eta_{\alpha}\left(R\left(U, \xi_{\alpha}\right) W\right)+\eta_{\alpha}(V) \eta_{\alpha}(R(U, Y) W)-g(Y, W) \eta_{\alpha}\left(R(U, V) \xi_{\alpha}\right)+\eta_{\alpha}(W) \eta_{\alpha}(R(U, V) Y)\right\}=0 .
\end{aligned}
$$

By using (4.2), (4.4) in (5.3) we have

$$
R(U, V, W, Y)=s\{g(Y, U) g(V, W)-g(Y, V) g(U, W)\} .
$$

Taking $U=Y=e_{i}$ in (5.4) and summing over $i=1,2, \ldots \ldots .2 n+s$ we get

$$
S(V, W)=s(2 n+s-1) g(V, W)
$$

Thus we state the following;

Theorem $5 S$-manifold satisfying the condition $C \cdot R=0$ is an Einstein manifold.

From (5.5) and (3.9) we have

$$
(s(2 n+s-1)+\lambda) g(X, Y)=0(5.6)
$$

Taking $X=Y=e_{i}$ in (5.6) and summing over $i=1,2, \ldots \ldots .2 n+s$, we get the value of $\lambda$

$$
\lambda=-s(2 n+s-1)(<0)
$$

Thus we state the following;

Theorem 6 Ricci soliton in $S$-manifold satisfying the condition $C \cdot R=0$ is shrinking. 
Corollary 3 Ricci soliton in $S$-manifold satisfying $C \cdot R=0$ is steady if $s=0$ (Kaehler manifold) and is shrinking if $s=1$ (Sasakian manifold).

Ricci soliton in $S$-manifolds satisfying $C \cdot C=0$.

Let us assume that the condition $C\left(\left(\xi_{\alpha}, Y\right) \cdot C\right)(U, V) W=0$ hold on $M$, then $C\left(\xi_{\alpha}, Y\right) C(U, V) W-C\left(C\left(\xi_{\alpha}, Y\right) U, V\right) W-C\left(U, C\left(\xi_{\alpha}, Y\right) V\right) W-C(U, V) C\left(\xi_{\alpha}, Y\right) W=0$.

Using (4.3) in (6.1), we get

$$
\begin{array}{r}
{\left[s-\frac{r}{2 n(2 n+1)}\right]_{\alpha}\left\{g(Y, C(U, V) W) \xi_{\alpha}-\eta_{\alpha}(C(U, V) W) Y-g(Y, U) C\left(\xi_{\alpha}, V\right) W+\eta_{\alpha}(U) C(Y, V) W\right.} \\
\left.-g(Y, V) C\left(U, \xi_{\alpha}\right) W+\eta_{\alpha}(V) C(U, Y) W-g(Y, W) C(U, V) \xi_{\alpha}+\eta_{\alpha}(W) C(U, V) Y\right\}=0
\end{array}
$$

By taking an inner product with $\xi_{\alpha}$ then we get

$$
\begin{aligned}
& \sum_{\alpha}\left\{s C(U, V, W, Y)-\eta_{\alpha}(C(U, V) W) \eta_{\alpha}(Y)-g(Y, U) \eta_{\alpha}\left(C\left(\xi_{\alpha}, V\right) W\right)+\eta_{\alpha}(U) \eta_{\alpha}(C(Y, V) W)\right. \\
& \left.-g(Y, V) \eta_{\alpha}\left(C\left(U, \xi_{\alpha}\right) W\right)+\eta_{\alpha}(V) \eta_{\alpha}(C(U, Y) W)-g(Y, W) \eta_{\alpha}\left(C(U, V) \xi_{\alpha}\right)+\eta_{\alpha}(W) \eta_{\alpha}(C(U, V) Y)\right\}=0 .
\end{aligned}
$$

By using (4.2), (4.4) in (6.3) we have

$$
C(U, V, W, Y)=\left[s-\frac{r}{2 n(2 n+1)}\right]\{g(Y, U) g(V, W)-g(Y, V) g(U, W)\} .
$$

Taking $U=Y=e_{i}$ in (4.8) and summing over $i=1,2, \ldots \ldots . .2 n+s$ and using (4.1) we get

$$
S(V, W)=s(2 n+s-1) g(V, W)
$$

Thus we state the following;

Theorem $7 S$-manifold satisfying the condition $C \cdot C=0$ is an Einstein manifold.

From (6.5) and (3.9) we have

$$
(s(2 n+s-1)+\lambda) g(X, Y)=0(6.6)
$$

Taking $X=Y=e_{i}$ in (6.6) and summing over $i=1,2, \ldots \ldots .2 n+s$, we get the value of $\lambda$

$$
\lambda=-s(2 n+s-1)(<0)
$$

Thus we state the following;

Theorem 8 Ricci soliton in $S$-manifold satisfying the condition $C \cdot C=0$ is shrinking.

Corollary 4 Ricci soliton in $S$-manifold satisfying $C \cdot C=0$ is steady if $s=0$ (Kaehler manifold) and is shrinking if $s=1$ (Sasakian manifold).

\section{Ricci soliton in Pseudo-symmetric $S$-manifolds}

An $S$-manifold is said to be Pseudo-symmetric if $R \cdot R=L_{1} Q(g, R)$.

$$
\begin{aligned}
& \left(R\left(\xi_{\alpha}, Y\right) \cdot R\right)(U, V) W=L_{1}\left[\left(\left(\xi_{\alpha} \wedge Y\right) \cdot R\right)(U, V) W\right], \quad \text { (7.1) } \\
& R\left(\xi_{\alpha}, Y\right) R(U, V) W-R\left(R\left(\xi_{\alpha}, Y\right) U, V\right) W-R\left(U, R\left(\xi_{\alpha}, Y\right) V\right) W-R(U, V) R\left(\xi_{\alpha}, Y\right) W \\
& =L_{1}\left[\left(\xi_{\alpha} \wedge Y\right) R(U, V) W-R\left(\left(\xi_{\alpha} \wedge Y\right) U, V\right) W-R\left(U,\left(\xi_{\alpha} \wedge Y\right) V\right) W-R(U, V)\left(\xi_{\alpha} \wedge Y\right) W\right]
\end{aligned}
$$

Using (2.11) L.H.S of (??) is 


$$
\begin{aligned}
& s \sum_{\alpha}\left\{g(Y, R(U, V) W) \xi_{\alpha}-\eta_{\alpha}(R(U, V) W) Y-g(Y, U) R\left(\xi_{\alpha}, V\right) W+\eta_{\alpha}(U) R(Y, V) W\right. \\
& \left.-g(Y, V) R\left(U, \xi_{\alpha}\right) W+\eta_{\alpha}(V) R(U, Y) W-g(Y, W) R(U, V) \xi_{\alpha}+\eta_{\alpha}(W) R(U, V) Y\right\} .
\end{aligned}
$$

By taking an inner product with $\xi_{\alpha}$ then we get

$s \sum_{\alpha}\left\{s R(U, V, W, Y)-\eta_{\alpha}(R(U, V) W) \eta_{\alpha}(Y)-g(Y, U) \eta_{\alpha}\left(R\left(\xi_{\alpha}, V\right) W\right)+\eta_{\alpha}(U) \eta_{\alpha}(R(Y, V) W)\right.$

$\left.-g(Y, V) \eta_{\alpha}\left(R\left(U, \xi_{\alpha}\right) W\right)+\eta_{\alpha}(V) \eta_{\alpha}(R(U, Y) W)-g(Y, W) \eta_{\alpha}\left(R(U, V) \xi_{\alpha}\right)+\eta_{\alpha}(W) \eta_{\alpha}(R(U, V) Y)\right\}$.

By using (2.10), (2.12) in (??) we have

$s\left\{s R(U, V, W, Y)+s^{2} g(Y, V) g(U, W)-s^{2} g(Y, U) g(V, W)\right\}$.

Again using (2.11) R.H.S of (??), we get

$$
\begin{aligned}
& L_{1}\left[\sum _ { \alpha } \left\{g(Y, R(U, V) W) \xi_{\alpha}-\eta_{\alpha}(R(U, V) W) Y-g(Y, U) R\left(\xi_{\alpha}, V\right) W+\eta_{\alpha}(U) R(Y, V) W\right.\right. \\
& \left.\left.-g(Y, V) R\left(U, \xi_{\alpha}\right) W+\eta_{\alpha}(V) R(U, Y) W-g(Y, W) R(U, V) \xi_{\alpha}+\eta_{\alpha}(W) R(U, V) Y\right\}\right] .
\end{aligned}
$$

By taking an inner product with $\xi_{\alpha}$ then we get

$$
\begin{aligned}
& L_{1}\left[\sum _ { \alpha } \left\{s R(U, V, W, Y)-\eta_{\alpha}(R(U, V) W) \eta_{\alpha}(Y)-g(Y, U) \eta_{\alpha}\left(R\left(\xi_{\alpha}, V\right) W\right)+\eta_{\alpha}(U) \eta_{\alpha}(R(Y, V) W)\right.\right. \\
& \left.\left.-g(Y, V) \eta_{\alpha}\left(R\left(U, \xi_{\alpha}\right) W\right)+\eta_{\alpha}(V) \eta_{\alpha}(R(U, Y) W)-g(Y, W) \eta_{\alpha}\left(R(U, V) \xi_{\alpha}\right)+\eta_{\alpha}(W) \eta_{\alpha}(R(U, V) Y)\right\}\right] .
\end{aligned}
$$

By using (2.10), (2.12) in (7.7) we have

$$
L_{1}\left[s R(U, V, W, Y)+s^{2} g(Y, V) g(U, W)-s^{2} g(Y, U) g(V, W)\right]
$$

From (7.5) and (7.8) we get

$$
\left[L_{1}-s\right]\left[s R(U, V, W, Y)+s^{2} g(Y, V) g(U, W)-s^{2} g(Y, U) g(V, W)\right]=0 .
$$

Therefore either $L_{1}=s$ or

$$
R(U, V, W, Y)=s\{g(Y, U) g(V, W)-g(Y, V) g(U, W)\} .
$$

Taking $U=Y=e_{i}$ in (7.10) and summing over $i=1,2, \ldots \ldots . .2 n+s$ we get

$$
S(V, W)=s(2 n+s-1) g(V, W)
$$

Thus we state the following;

Theorem 9 Pseudo symmetric $S$-manifold is an Einstein manifold provided $L_{1} \neq S$

From (7.11) and (3.9) we have

$$
(s(2 n+s-1)+\lambda) g(X, Y)=0(7.12)
$$

Taking $X=Y=e_{i}$ in (7.12) and summing over $i=1,2, \ldots \ldots .2 n+s$, we get the value of $\lambda$

$$
\lambda=-s(2 n+s-1)(<0)
$$

Thus we state the following;

Theorem 10 Ricci soliton in pseudo symmetric $S$-manifold is shrinking.

Corollary 5 Ricci soliton in pseudo symmetric $S$-manifold is steady if $s=0$ (Kaehler manifold) and is shrinking if $s=1$ (Sasakian manifold).

VII. Ricci soliton in $S$-manifolds satisfying $R \cdot C=L_{2} Q(g, C)$.

Let us assume that the condition $R\left(\left(\xi_{\alpha}, Y\right) \cdot C\right)(U, V) W=L_{2}\left[\left(\xi_{\alpha} \wedge Y\right) \cdot C\right](U, V) W$ hold on $M$, then

$$
\begin{aligned}
& R\left(\xi_{\alpha}, Y\right) C(U, V) W-C\left(R\left(\xi_{\alpha}, Y\right) U, V\right) W-C\left(U, R\left(\xi_{\alpha}, Y\right) V\right) W-C(U, V) R\left(\xi_{\alpha}, Y\right) W \\
& =L_{2}\left[\left(\xi_{\alpha} \wedge Y\right) C(U, V) W-C\left(\left(\xi_{\alpha} \wedge Y\right) U, V\right) W-C\left(U,\left(\xi_{\alpha} \wedge Y\right) V\right) W-C(U, V)\left(\xi_{\alpha} \wedge Y\right) W\right]
\end{aligned}
$$


(8.1)

Using (2.11) L.H.S of (??) is

$$
\begin{aligned}
& s \sum_{\alpha}\left\{g(Y, C(U, V) W) \xi_{\alpha}-\eta_{\alpha}(C(U, V) W) Y-g(Y, U) C\left(\xi_{\alpha}, V\right) W+\eta_{\alpha}(U) C(Y, V) W\right. \\
& \left.-g(Y, V) C\left(U, \xi_{\alpha}\right) W+\eta_{\alpha}(V) C(U, Y) W-g(Y, W) C(U, V) \xi_{\alpha}+\eta_{\alpha}(W) C(U, V) Y\right\} .
\end{aligned}
$$

By taking an inner product with $\xi_{\alpha}$ then we get

$$
\begin{aligned}
& s \sum_{\alpha}\left\{s C(U, V, W, Y)-\eta_{\alpha}(C(U, V) W) \eta_{\alpha}(Y)-g(Y, U) \eta_{\alpha}\left(C\left(\xi_{\alpha}, V\right) W\right)+\eta_{\alpha}(U) \eta_{\alpha}(C(Y, V) W)\right. \\
& \left.-g(Y, V) \eta_{\alpha}\left(C\left(U, \xi_{\alpha}\right) W\right)+\eta_{\alpha}(V) \eta_{\alpha}(C(U, Y) W)-g(Y, W) \eta_{\alpha}\left(C(U, V) \xi_{\alpha}\right)+\eta_{\alpha}(W) \eta_{\alpha}(C(U, V) Y)\right\} .
\end{aligned}
$$

By using (4.2), (4.4) in (??) we have

$$
s^{2}\left\{C(U, V, W, Y)-\left[s-\frac{r}{2 n(2 n+1)}\right][g(Y, U) g(V, W)-g(Y, V) g(U, W)]\right\} .
$$

Again using (2.11) R.H.S of (8.1) is

$$
\begin{aligned}
& L_{2} \sum_{\alpha}\left\{g(Y, C(U, V) W) \xi_{\alpha}-\eta_{\alpha}(C(U, V) W) Y-g(Y, U) C\left(\xi_{\alpha}, V\right) W+\eta_{\alpha}(U) C(Y, V) W\right. \\
& \left.-g(Y, V) C\left(U, \xi_{\alpha}\right) W+\eta_{\alpha}(V) C(U, Y) W-g(Y, W) C(U, V) \xi_{\alpha}+\eta_{\alpha}(W) C(U, V) Y\right\} .(8.5)
\end{aligned}
$$

By taking an inner product with $\xi_{\alpha}$ then we get

$$
\begin{aligned}
& L_{2} \sum_{\alpha}\left\{s C(U, V, W, Y)-\eta_{\alpha}(C(U, V) W) \eta_{\alpha}(Y)-g(Y, U) \eta_{\alpha}\left(C\left(\xi_{\alpha}, V\right) W\right)+\eta_{\alpha}(U) \eta_{\alpha}(C(Y, V) W)\right. \\
& \left.-g(Y, V) \eta_{\alpha}\left(C\left(U, \xi_{\alpha}\right) W\right)+\eta_{\alpha}(V) \eta_{\alpha}(C(U, Y) W)-g(Y, W) \eta_{\alpha}\left(C(U, V) \xi_{\alpha}\right)+\eta_{\alpha}(W) \eta_{\alpha}(C(U, V) Y)\right\} .
\end{aligned}
$$

By using (4.2), (4.4) in (8.6) we have

$$
s L_{2}\left\{C(U, V, W, Y)-\left[s-\frac{r}{2 n(2 n+1)}\right][g(Y, U) g(V, W)-g(Y, V) g(U, W)]\right\}
$$

From (8.4) and (8.7) we get

$$
\left[s L_{2}-s^{2}\right]\left\{C(U, V, W, Y)-\left[s-\frac{r}{2 n(2 n+1)}\right][g(Y, U) g(V, W)-g(Y, V) g(U, W)]\right\}=0
$$

Therefore either $L_{2}=s$ or

$$
C(U, V, W, Y)=\left[s-\frac{r}{2 n(2 n+1)}\right][g(Y, U) g(V, W)-g(Y, V) g(U, W)]
$$

Taking $U=Y=e_{i}$ in (8.9) and summing over $i=1,2, \ldots \ldots .2 n+s$ we get

$$
S(V, W)=s(2 n+s-1) g(V, W)
$$

Thus we state the following;

Theorem $11 S$-manifold satisfying the condition $R \cdot C=L_{2} Q(g, C)$ is an Einstein manifold provided $L_{2} \neq s$.

From (8.10) and (3.9) we have

$$
(s(2 n+s-1)+\lambda) g(X, Y)=0(8.11)
$$

Taking $X=Y=e_{i}$ in (8.11) and summing over $i=1,2, \ldots \ldots .2 n+s$, we get the value of $\lambda$

$$
\lambda=-s(2 n+s-1)(<0)
$$

Thus we state the following; 
Theorem 12 Ricci soliton in $S$-manifold satisfying the condition $R \cdot C=L_{2} Q(g, C)$ is shrinking.

Corollary 6 Ricci soliton in $S$-manifold satisfying $R \cdot C=L_{2} Q(g, C)$ is steady if $s=0$ (Kaehler manifold) and is shrinking if $s=1$ (Sasakian manifold).

VIII. Ricci soliton in $S$-manifolds satisfying $C \cdot R=L_{3} Q(g, R)$.

Let us assume that the condition $C\left(\left(\xi_{\alpha}, Y\right) \cdot R\right)(U, V) W=L_{3}\left[\left(\xi_{\alpha} \wedge Y\right) \cdot R\right](U, V) W$ hold on $M$, then

$$
C\left(\xi_{\alpha}, Y\right) R(U, V) W-R\left(C\left(\xi_{\alpha}, Y\right) U, V\right) W-R\left(U, C\left(\xi_{\alpha}, Y\right) V\right) W-R(U, V) C\left(\xi_{\alpha}, Y\right) W
$$$$
=L_{3}\left[\left(\xi_{\alpha} \wedge Y\right) R(U, V) W-R\left(\left(\xi_{\alpha} \wedge Y\right) U, V\right) W-R\left(U,\left(\xi_{\alpha} \wedge Y\right) V\right) W-R(U, V)\left(\xi_{\alpha} \wedge Y\right) W\right]
$$

Using (5.2), (5.3), (7.6) and (7.7) in (9.1) we get

$$
\left\{s L_{3}-\left[s-\frac{r}{2 n(2 n+1)}\right]\right\}\{R(U, V, W, Y)-s[g(Y, U) g(V, W)-g(Y, V) g(U, W)]\}=0
$$

Therefore, either $L_{3}=s-\frac{r}{2 n(2 n+1)}$ or

$$
R(U, V, W, Y)=s\{g(Y, U) g(V, W)-g(Y, V) g(U, W)\} .
$$

Taking $U=Y=e_{i}$ in (9.3) and summing over $i=1,2, \ldots \ldots .2 n+s$ we get

$$
S(V, W)=s(2 n+s-1) g(V, W)
$$

Thus we state the following;

Theorem $13 S$-manifold satisfying the condition $C \cdot R=L_{3} Q(g, R)$ is an Einstein manifold provided

$$
L_{3} \neq s-\frac{r}{2 n(2 n+1)} \text {. }
$$

From (9.4) and (3.9) we have

$$
(s(2 n+s-1)+\lambda) g(X, Y)=0(9.5)
$$

Taking $X=Y=e_{i}$ in (9.5) and summing over $i=1,2, \ldots \ldots . .2 n+s$, we get the value of $\lambda$

$$
\lambda=-s(2 n+s-1)(<0)
$$

Thus we state the following;

Theorem 14 Ricci soliton in $S$-manifold satisfying the condition $C \cdot R=L_{3} Q(g, R)$ is shrinking.

Corollary 7 Ricci soliton in $S$-manifold satisfying $C \cdot R=L_{3} Q(g, R)$ is steady if $s=0$ (Kaehler manifold) and is shrinking if $s=1$ (Sasakian manifold).

\section{Ricci soliton in $S$-manifolds satisfying $C \cdot C=L_{4} Q(g, C)$.}

Let us assume that the condition $C\left(\left(\xi_{\alpha}, Y\right) \cdot C\right)(U, V) W=L_{4}\left[\left(\xi_{\alpha} \wedge Y\right) \cdot C\right](U, V) W$ hold on $M$, then

$$
\begin{aligned}
& C\left(\xi_{\alpha}, Y\right) C(U, V) W-C\left(C\left(\xi_{\alpha}, Y\right) U, V\right) W-C\left(U, C\left(\xi_{\alpha}, Y\right) V\right) W-C(U, V) C\left(\xi_{\alpha}, Y\right) W \\
& =L_{4}\left[\left(\xi_{\alpha} \wedge Y\right) C(U, V) W-C\left(\left(\xi_{\alpha} \wedge Y\right) U, V\right) W-C\left(U,\left(\xi_{\alpha} \wedge Y\right) V\right) W-C(U, V)\left(\xi_{\alpha} \wedge Y\right) W\right] \\
& (10.1)
\end{aligned}
$$

Using (6.2), (6.3), (8.5) and (8.6) in (10.1) we get

$$
\left\{s L_{4}-\left[s-\frac{r}{2 n(2 n+1)}\right]\right\}\left\{C(U, V, W, Y)-\left[s-\frac{r}{2 n(2 n+1)}\right][g(Y, U) g(V, W)-g(Y, V) g(U, W)]\right\}=0
$$


Therefore, either $L_{4}=s-\frac{r}{2 n(2 n+1)}$ or

$$
C(U, V, W, Y)=\left[s-\frac{r}{2 n(2 n+1)}\right]\{g(Y, U) g(V, W)-g(Y, V) g(U, W)\} .
$$

Taking $U=Y=e_{i}$ in (10.3) and summing over $i=1,2, \ldots \ldots .2 n+s$, using (4.1) we get

$$
S(V, W)=s(2 n+s-1) g(V, W)
$$

Thus we state the following;

Theorem $15 S$-manifold satisfying the condition $C \cdot C=L_{4} Q(g, C)$ is an Einstein manifold provided

$$
L_{4} \neq s-\frac{r}{2 n(2 n+1)} \text {. }
$$

From (10.4) and (3.9) we have

$$
(s(2 n+s-1)+\lambda) g(X, Y)=0(10.5)
$$

Taking $X=Y=e_{i}$ in (10.5) and summing over $i=1,2, \ldots \ldots .2 n+s$, we get the value of $\lambda$

$$
\lambda=-s(2 n+s-1)(<0)
$$

Thus we state the following;

Theorem 16 Ricci soliton in $S$-manifold satisfying the condition $C \cdot C=L_{4} Q(g, C)$ is shrinking.

Corollary 8 Ricci soliton in $S$-manifold satisfying $C \cdot C=L_{4} Q(g, C)$ is steady if $s=0$ (Kaehler manifold) and is shrinking if $s=1$ (Sasakian manifold).

\section{Conclusion}

It is shown that Ricci soliton in $S$-manifold satisfying semi-symmetric and pseudo-symmetric conditions are shrinking. Hence if $S=1$, then Sasakian manifolds are shrinking which in accordance with [1], [5], [14], and if $S=0$, then Kaehler manifolds are steady [21]

\section{References}

[1]. C. S. Bagewadi and G. Ingalahalli, Ricci solitons in Lorentzian $\alpha$-Sasakian manifolds, Acta Mathematica Academiae Paedagogicae Nyiregyhaziensis, vol. 28, no.1, pp. 59-68,2012.

[2]. C. S. Bagewadi and K.R.Vidyavathi, Ricci soliton of almost $C(\alpha)$ manifolds, Bull. Cal. Math. Soc., 107,(6) 483-494 (2015) .

[3]. D.E. Blair, Geometry of manifolds with structural group $U(n) \times O(s)$, J. Differential Geom. 4 (1970), 155-167

[4]. D.E. Blair, G. Ludden and K. Yano., Differential geometric structures on principal toroidal bundles, Trans. Amer. Math. Soc. 181 (1973), 175-184.

[5]. Chenxu He and Meng Zhu Ricci solitons on Sasakian manifolds, Arxiv:1109.4407v2 [math.DG]

[6]. C. C $\breve{a}$ lin and M. Crasmareanu, From the Eisenhart problem to Ricci solitons in f-Kenmotsu manifolds, Bulletin of the Malaysian Mathematical Sciences Society, vol. 33, no.3, pp. 367-368, 2010.

[7]. S. Debnath and A. Battacharya, Second order parallel tensor in Trans-Sasakian Manifolds and connection with Ricci soliton, Lobachevski Journal of Mathematics, Vol. 33, No. 4, (2012), 312-316.

[8]. R. Deszcz, On pseudosymmetric spaces, Bull. Soc. Math. Belg. Ser.A,44, 1(1992), 319-324.

[9]. R. Deszcz and W. Gryeak, On some class of wraped product manifolds, Bull. Inst. Math. Acad. Sinica 51, 311(1987).

[10]. L.P. Eisenhart, Symmetric tensors of the second order whose first covariant serivates are zero, Trans. Amer. Math. Soc. 25, no-2 (1923), 297-306.

[11]. S.I. Goldberg and K. Yano, On normal globally framed $f$-manifolds, Tohoku Math. J. 22 (1970), 362-370.

[12]. S.I. Goldberg and K. Yano, Globally framed $f$-manifolds, Illinois J. Math. 15 (1971), 456-474.

[13]. I. Hasegawa, Y. Oknyama and T. Abe, On the p-th Sasakian manifolds, J. Hokkaido Univ. Ed. SectII A 37(1) (1986), 1-16.

[14]. G. Ingalahalli and C. S. Bagewadi, Ricci solitons in $\alpha$-Sasakain manifolds, ISRN Geometry, vol. 2012, Article ID 421384, 14 pages, 2012.

[15]. S. Ishihara, Normal structure $f$ satisfying $f_{3}+f=0$, Kodai Math. Sem. Rep. 18 (1966), 36-47.

[16]. H. Nakagawa, $f$-structures induced on submanifolds in spaces, almost Hermitian or Kaehlerian, Kodai Math. Sem. Rep. 18 (1966), 161-183.

[17]. H. Nakagawa, On framed $f$-manifolds, Kodai Math. Sem. Rep. 18 (1966), 293-306.

[18]. K. Nomizu, On hypersurface satisfying a certain condition on the curvature tensor, Tohoku Math. J., 20, 45(1968).

[19]. Y.A. Ogawa, Conditions for a compact Kaehalerian space to be locally symmetric, Natur. Sci. Report, Ochanomizu Univ. 28,21(1977) 
[20]. D. Perreone, Contact Riemannian manifolds satisfying $R(\xi, X) R=0$, Yokohama math. J., 39(2)(1992), 141-149.

[21]. M. M. Praveena and C. S. Bagewadi, On almost pseudo Bochner symmetric generalized complex space forms, Acta Mathematica Academiae Paedagogicae Nyiregyhaziensis, 32(2016), 149-159.

[22]. R. Sharma, Second order parallel tensor in real and complex space forms, International J. Math and Math. Sci., 12 (1989), 787-790.

[23]. R. Sharma, Second order parallel tensor on contact manifolds, Algebra, Groups and Geometries, 7 (1990), 787-790.

[24]. R. Sharma, Second order parallel tensor on contact manifolds II, C.R. Math Rep. Acad. Sci. Canada XIII, No-6, 6 (1991), $259-264$.

[25]. Z.I. Szabo, Structure theorems on Riemannian spaces satiesfying $R(X, Y) \cdot R=0$, I, The local version, J. Differential Geom. 17, 531(1982).

[26]. S. Tanno, Locally symmetric K-contact Riemannian manifolds, Proc. Japan Acad. 43, 581(1967).

[27]. M. M. Tripathi, Ricci solitons in contact metric manifolds, http://arxiv.org/abs/0801.4222.

[28]. K. Yano, On a structure defined by a tensor field $f$ of type $(1,1)$ satisfying $f_{3}+f=0$, Tensor N.S. 14 (1963), 99-109. 